\title{
Subgame-perfection in free transition games
}

Citation for published version (APA):

Flesch, J., Kuipers, J., Schoenmakers, G., \& Vrieze, K. (2011). Subgame-perfection in free transition games. METEOR, Maastricht University School of Business and Economics. METEOR Research Memorandum No. 047 https://doi.org/10.26481/umamet.2011047

Document status and date:

Published: 01/01/2011

DOI:

10.26481/umamet.2011047

Document Version:

Publisher's PDF, also known as Version of record

\section{Please check the document version of this publication:}

- A submitted manuscript is the version of the article upon submission and before peer-review. There can be important differences between the submitted version and the official published version of record.

People interested in the research are advised to contact the author for the final version of the publication, or visit the DOI to the publisher's website.

- The final author version and the galley proof are versions of the publication after peer review.

- The final published version features the final layout of the paper including the volume, issue and page numbers.

Link to publication

\footnotetext{
General rights rights.

- You may freely distribute the URL identifying the publication in the public portal. please follow below link for the End User Agreement:

www.umlib.nl/taverne-license

Take down policy

If you believe that this document breaches copyright please contact us at:

repository@maastrichtuniversity.nl

providing details and we will investigate your claim.
}

Copyright and moral rights for the publications made accessible in the public portal are retained by the authors and/or other copyright owners and it is a condition of accessing publications that users recognise and abide by the legal requirements associated with these

- Users may download and print one copy of any publication from the public portal for the purpose of private study or research.

- You may not further distribute the material or use it for any profit-making activity or commercial gain

If the publication is distributed under the terms of Article $25 \mathrm{fa}$ of the Dutch Copyright Act, indicated by the "Taverne" license above, 


\section{Maastricht University}

J. Flesch, J. Kuipers,

G. Schoenmakers, K. Vrieze

Subgame-Perfection in Free Transition Games

$\mathrm{RM} / 11 / 047$

\section{METEOR}

Maastricht University School of Business and Economics

Maastricht Research School of Economics

of Technology and Organization

P.O. Box 616

NL - 6200 MD Maastricht

The Netherlands 


\title{
Subgame-Perfection in Free Transition Games
}

\author{
J. Flesch, J. Kuipers, G. Schoenmakers ${ }^{\dagger}$, K. Vrieze $^{\dagger}$
}

October 11, 2011

\begin{abstract}
We prove the existence of a subgame-perfect $\varepsilon$-equilibrium, for every $\varepsilon>0$, in a class of multi-player games with perfect information, which we call free transition games. The novelty is that a non-trivial class of perfect information games is solved for subgameperfection, with multiple non-terminating actions, in which the payoff structure is generally not semi-continuous. Due to the lack of semi-continuity, there is no general rule of comparison between the payoffs that a player can obtain by deviating a large but finite number of times or, respectively, infinitely many times. We introduce new techniques to overcome this difficulty.

Our construction relies on an iterative scheme which is independent of $\varepsilon$ and terminates in polynomial time with the following output: for all possible histories $h$, a pure action $a_{h}^{1}$ or in some cases two pure actions $a_{h}^{2}$ and $b_{h}^{2}$ for the active player at $h$. The subgame-perfect $\varepsilon$-equilibrium then prescribes for every history $h$ that the active player plays $a_{h}^{1}$ with probability 1 or respectively plays $a_{h}^{2}$ with probability $1-\delta(\varepsilon)$ and $b_{h}^{2}$ with probability $\delta(\varepsilon)$. Here, $\delta(\varepsilon)$ is arbitrary as long as it is positive and small compared to $\varepsilon$, so the strategies can be made "almost" pure.

Keywords: perfect information game, recursive game, subgame-perfect equilibrium, average payoff.
\end{abstract}

\section{Introduction}

In many practical situations, decision makers have to make a number of choices sequentially, with perfect knowledge of all past decisions. To model and to analyse such situations, the mathematical framework of non-cooperative games with perfect information has served as a useful tool. In those games, a widely accepted solution concept is the one of Nash equilibrium, which is a collection of strategies, one for each player, such that no player can profit by individually deviating from his strategy. In other words, in a Nash equilibrium, every player plays a strategy that is a best response to the strategies of his opponents. Yet, if a player or some players make choices, either on purpose or by mistake, that differ from the prescriptions of their strategies, the continuation strategies may no longer enjoy the above mentioned equilibrium property in the remaining subgame. Hence,

*Department of Quantitative Economics, Maastricht University, P.O. Box 616, 6200MD, Maastricht, The Netherlands.

${ }^{\dagger}$ Department of Knowledge Engineering, Maastricht University, P.O. Box 616, 6200MD, Maastricht, The Netherlands. 
in certain cases the strategies may prescribe non-credible behavior for the players, who consequently may become reluctant to follow them. Realizing this shortcoming, Selten [13] and [14] proposed the notion of a subgame-perfect (Nash) equilibrium, as a selection criterion amongst the Nash equilibria of the game. A subgame-perfect equilibrium requires that, in every subgame, the continuation strategies must form a Nash equilibrium. Due to the lack of existence of such exact solutions, we often need to allow for a positive error term $\varepsilon$. While, in fairly general perfect information games, the existence of a Nash $\varepsilon$-equilibrium, for every $\varepsilon>0$, has long been known due to Mertens and Neyman, cf. Mertens [9], and later to Thuijsman and Raghavan [18], Flesch et al. [5] have recently shown that not all perfect information games admit a subgame-perfect $\varepsilon$-equilibrium. It is still an open question which classes of perfect information games do.

Given the large number and the diversity of applications of subgame-perfect equilibrium, also in perfect information games, this existence question is relevant for both theory and practice, and has received notable attention in recent years. Areas where subgame-perfect equilibria play an important role include bargaining (cf. e.g. Osborne and Rubinstein [11], Muthoo [10], Weg and Zwick [20]), coalition formation (cf. Bloch [1], Vartiainen [19]), entry deterrence (cf. Selten [15]) and political science (cf. Bueno de Mesquita and Lalman [2]).

In this paper we examine a natural class of perfect information games, which we call free transition games, and prove that they admit a subgame-perfect $\varepsilon$-equilibrium for all $\varepsilon>0$. Moreover, the corresponding strategies are "almost" pure: either one action is prescribed with probability 1 , or one action with probability $1-\delta$ and another one with probability $\delta$, where $\delta>0$ is arbitrarily small.

A free transition game is played as follows: at every period during play, one of the players is called active. The active player can either terminate the game, or he can announce any other player, who then becomes the active player. If a player decides to terminate the game, then depending on the identity of this player, every player receives a payoff. If no player ever terminates the game, then every player receives payoff zero.

One could think of a group of friends, who want to spend the evening together. Initially, one of them, say person $j$, is supposed to organize the evening activities. He can either organize the evening himself (termination at $j$ ), or ask someone else, say person $k$, to replace him as the organizer. In the latter case, person $k$ has the following options: organize the evening (termination at $k$ ), reject person $j$ 's request (person $k$ nominates $j$ ), or ask a third person to replace him as the organizer. In the last two cases, the procedure continues as before. Since the evening activities may depend on the organizer, the payoff of each person may depend on the identity of the organizer. If no one ever decides to organize the evening, the friends cannot meet and they all receive payoff zero.

We remark that such a game can be viewed as a special type of multi-player stochastic game with the following properties: (1) it is a game with perfect information (i.e., in every state, only one of the players is to choose an action), (2) the payoffs are recursive (i.e., the only payoffs are upon termination), (3) all the transitions are deterministic.

In this paper we prove that every free transition game admits a subgame-perfect $\varepsilon$ equilibrium for every $\varepsilon>0$. In view of an instructive example in Solan \& Vieille [17], this result cannot be strengthened to pure strategies or to $\varepsilon=0$. Our construction uses 
an iterative method that results in a non-empty collection of plays that can be a part of a subgame-perfect $\varepsilon$-equilibrium, for every $\varepsilon>0$. The main difficulty arises by negative termination payoffs, which may dissuade certain players from terminating the game.

For free transition games with only non-negative termination payoffs, the one-deviation principle applies, cf. Kuipers et al. [6]: if with respect to a strategy profile, no player can improve his payoff in any subgame by deviating exactly once, then this strategy profile is a subgame-perfect 0-equilibrium. Indeed, this property implies that a finite number of deviations cannot be profitable either, and because the termination payoffs are nonnegative, no player can profit from deviating infinitely many times (that can only happen if play never terminates). By making use of this one-deviation principle, Kuipers et al. [6] showed the existence of a pure subgame-perfect 0-equilibrium.

Within the more general framework of perfect-information games, there has been significant development in recent years on the existence of subgame-perfect $\varepsilon$-equilibria. Solan [16], Mashiah-Yaakovi [7] and [8] proved the existence of (approximate) subgame-perfect $\varepsilon$-equilibria in different subclasses in which the active player has only one non-terminating action. When multiple non-terminating actions are allowed, the game becomes strategically more complex. Iterative methods have been proven useful in a number of subclasses. Kuipers et al. [6] and Flesch et al. [3] applied such methods, but in both cases it is crucial to assume that the termination payoffs are non-negative. These results have been generalized by Flesch et al. [4], who proved the existence of subgame-perfect $\varepsilon$-equilibria, provided that the transitions are deterministic and that the payoff functions of the players, defined as a function of the whole play, are bounded and lower-semicontinuous. The assumption of lower-semicontinuity guarantees that deviating infinitely many times is never better, up to a small error-term, than deviating a large but finite number of times, and hence the one-deviation principle still applies. Subsequently, Purves \& Sudderth [12] proved the same result, by using a different approach, when upper-semicontinuity is assumed instead. Due to upper-semicontinuity, the opposite is true here: deviating a large but finite number of times is never better, up to a small error-term, than deviating infinitely many times.

Our model of free transition games, with possibly negative termination payoffs, differs from the above mentioned classes with multiple non-terminating actions, because the payoff functions are generally not semi-continuous. Consequently, there is no general rule of comparison between deviations finitely many respectively infinitely many times, and this issue requires a delicate treatment.

\section{Game model and strategic concepts}

A free transition game is given by a triple $G_{j}=(N, r, j)$, where $N=\{1, \ldots, n\}$ is a set of players with $n \geq 2, j \in N$, and $r: N \rightarrow \mathbb{R}^{n}$ is a function that associates a payoff vector $r(t) \in \mathbb{R}^{n}$ with each player $t \in N$. The game is played at periods in $\mathbb{N}$ as follows. At every period, one of the players is called active, with $j$ being the active player at period 1. The active player, say $t$, always has the option to terminate the game. In this case, the players receive payoffs according to $r(t)$. The active player can also announce any player

other than himself, who then becomes the active player at the next period, and so on. If no player ever terminates the game, each player's payoff is zero. 
Note that the set of states in a free transition game is exactly the set of players $N$. We will use the term player or state depending on the context.

For convenience, we assume that (i) the active player can terminate the game by announcing himself as the next active player, and (ii) if he does so, then play goes on, but this player has to announce himself at all further periods. So, play is always infinite and results in a sequence $\left(t_{m}\right)_{m \in \mathbb{N}}$ of active players such that if $t_{M}=t_{M+1}$ for some $M \in \mathbb{N}$ then $t_{m}=t_{M}$ for all $m \geq M$. If a player $s \in N$ announces himself, we say that termination occurs at $s$.

The history at some period is the sequence that consists of the active players at all previous periods and the current active player, in chronological order. Note that every history in the game $G_{j}$ starts with $j$. For $G_{j}$, we denote by $H_{j}$ the set of all possible histories, and by $H_{j i}$ the set of all possible histories in which $i$ is the current active player. A strategy $\sigma_{i}$ for player $i \in N$ is a map that assigns to every history $h \in H_{j i}$ a probability distribution $\sigma_{i}(h)$ on $N$ such that if termination has occurred in $h$ at $i$ then $\sigma_{i}(h)$ places probability 1 on $i$. The interpretation is that $\sigma_{i}$ prescribes for player $i$ to choose the next active player according to $\sigma_{i}(h)$, after every $h \in H_{j i}$. A vector of strategies $\sigma=\left(\sigma_{i}\right)_{i \in N}$, one for each player, is called a strategy profile. For a strategy profile $\sigma$, we denote by $\phi_{i}(\sigma)$ the expected payoff for player $i \in N$ if every player $s \in N$ plays according to $\sigma_{s}$.

A strategy profile $\sigma$ is called a (Nash) $\varepsilon$-equilibrium in $G_{j}$, for some $\varepsilon \geq 0$, if no player can gain more than $\varepsilon$ by individually deviating from his strategy, i.e. for every player $i$ and every strategy $\widetilde{\sigma}_{i}$ of player $i$ we have

$$
\phi_{i}\left(\sigma_{1}, \ldots, \sigma_{i-1}, \widetilde{\sigma}_{i}, \sigma_{i+1}, \ldots, \sigma_{n}\right) \leq \phi_{i}(\sigma)+\varepsilon \text {. }
$$

Consider a history $h=\left(t_{m}\right)_{m=1}^{L} \in H_{j s}$. We denote by $H_{s i}^{h}$ the set of all histories $h^{\prime}=$ $\left(t_{m}^{\prime}\right)_{m=1}^{L^{\prime}} \in H_{s i}$ such that $\left(h, h^{\prime}\right):=\left(t_{1}, \ldots, t_{L-1}, t_{1}^{\prime}, \ldots, t_{L^{\prime}}^{\prime}\right)$ is a history in $H_{j i}$. Note that $H_{s i}^{h}$ is empty exactly when termination occurred in $h$ at $s$ and $i \neq s$. For a strategy $\sigma_{i}$, the continuation strategy $\sigma_{i}^{h}$ for $h$ is defined as the map that assigns to every history $h^{\prime} \in H_{s i}^{h}$ the probability distribution $\sigma_{i}\left(h, h^{\prime}\right)$. Intuitively, the recommendation by $\sigma_{i}^{h}$ after $h^{\prime}$ is just as if $h$ had happened before $h^{\prime}$.

A strategy profile $\sigma$ is a subgame-perfect $\varepsilon$-equilibrium in $G_{j}$, for some $\varepsilon \geq 0$, if for every history $h \in H_{j}$, with some final state $s$, the continuation strategy profile $\sigma^{h}=\left(\sigma_{i}^{h}\right)_{i \in N}$ is an $\varepsilon$-equilibrium in $G_{s}$, i.e. for every player $i$ and every strategy $\tilde{\sigma}_{i}$ of player $i$ we have

$$
\phi_{i}\left(\sigma_{1}^{h}, \ldots, \sigma_{i-1}^{h}, \widetilde{\sigma}_{i}^{h}, \sigma_{i+1}^{h}, \ldots, \sigma_{n}^{h}\right) \leq \phi_{i}\left(\sigma^{h}\right)+\varepsilon .
$$

A deterministic game-plan, or simply plan, is an infinite sequence $g=\left(t_{m}\right)_{m \in \mathbb{N}}$ with $t_{m} \in N$ for all $m \in \mathbb{N}$ and with the property

$$
\left[\exists M \in \mathbb{N}: t_{M}=t_{M+1}\right] \quad \Rightarrow \quad\left[\forall m \geq M: t_{m}=t_{M}\right] .
$$

If $t_{M}=t_{M+1}=s$ then $g$ corresponds to a plan or play where player $s$ eventually terminates the game. We say that plan $g$ terminates at $s$. Otherwise, i.e., if $t_{m} \neq t_{m+1}$ for all $m \in \mathbb{N}$, we say that $g$ is non-terminating, which corresponds to a plan or play, where no player ever terminates the game. We say that $g$ is a plan for $t$ if $t_{1}=t$. We write $t \in g$ if $m \in \mathbb{N}$ 
exists with $t_{m}=t$. If $t \in N$ is such that $t \neq t_{1}$, then the sequence $\left(t, t_{1}, t_{2}, \ldots\right)$ is also a plan, and we will denote this plan as $(t, g)$.

For every plan $g$, let $\phi(g)$ denote the corresponding payoff vector in $\mathbb{R}^{n}$. Formally, $\phi(g)=$ $r(t)$ if $g$ terminates at $t$ and $\phi(g)=(0, \ldots, 0)$ if $g$ does not terminate.

Define $\Omega$ as the set of real vectors $\alpha \in \mathbb{R}^{n}$ with $\alpha_{t} \geq r_{t}(t)$ for every $t \in N$. Now take $\alpha \in \Omega$. We say that a plan $g$ is $\alpha$-viable if $\phi_{t}(g) \geq \alpha_{t}$ for all $t \in g$. This means that, whenever play is in some state $t$, and play is according to $g$, the active player $t$ will receive a payoff of at least $\alpha_{t}$. For $t \in N$, let $V_{t}(\alpha)$ denote the set of plans for $t$ that are $\alpha$-viable. Say that $t \in N$ is $\alpha$-positive if $\alpha_{t}>0$ and that $t$ is $\alpha$-terminating if $\alpha_{t}=r_{t}(t)$. Say that $t$ is $\alpha$-safe at $u \in N \backslash\{t\}$ if $\phi_{t}(g) \geq \alpha_{t}$ for all $g \in V_{u}(\alpha)$. Say that $t$ is $\alpha$-vulnerable if $\alpha_{t} \leq 0$ and if at least one of the following holds:

V1. a plan $g \in V_{u}(\alpha)$ exists for some $u \in N \backslash\{t\}$ such that $g$ terminates at $t$ and there is $x \in g, x \neq t$, with $r_{t}(x) \leq \alpha_{t}$;

V2. an $\alpha$-terminating state $x \in N \backslash\{t\}$ exists with $r_{t}(x) \leq \alpha_{t}$.

The motivation behind this definition is that, if player $t$ is $\alpha$-vulnerable, then he can be persuaded to terminate by the threat of either switching to $g$ and terminating at $x$ (according to V1) or terminating at $x$ (according to V2).

The following lemma establishes some fairly intuitive relationships between the concepts we just introduced.

Lemma 1 Let $\alpha \in \Omega$ and let $t, u, s \in N$ with $u \neq t, s \neq t$, and $s \neq u$.

1. If $t$ is $\alpha$-safe at $u$ and $u$ is $\alpha$-safe at $s$, then $t$ is $\alpha$-safe at $s$.

2. If $\alpha_{t} \leq 0$ and $t$ is not $\alpha$-vulnerable, then $t$ is $\alpha$-safe at $s$.

Proof. Let $\alpha \in \Omega$ and let $t, u, s \in N$ with $u \neq t, s \neq t$, and $s \neq u$.

Proof of 1: Assume that $t$ is $\alpha$-safe at $u$ and that $u$ is $\alpha$-safe at $s$. To prove that $t$ is $\alpha$-safe at $s$, choose an arbitrary plan $g \in V_{s}(\alpha)$. Since $u$ is $\alpha$-safe at $s$, we have $\phi_{u}(g) \geq \alpha_{u}$, hence $(u, g) \in V_{u}(\alpha)$. Then, since $t$ is $\alpha$-safe at $u$, we have $\phi_{t}(u, g) \geq \alpha_{t}$, hence $\phi_{t}(g) \geq \alpha_{t}$. Since $g$ was chosen arbitrarily in $V_{s}(\alpha)$, it follows that $t$ is $\alpha$-safe at $s$.

Proof of 2: Assume that $\alpha_{t} \leq 0$ and that $t$ is not $\alpha$-vulnerable. Choose an arbitrary plan $g$ in $V_{s}(\alpha)$. If $g$ terminates at $t$, then the $\alpha$-viability of $g$ ensures that $\phi_{t}(g) \geq \alpha_{t}$. If $g$ terminates at $s^{\prime} \neq t$, then $r_{t}\left(s^{\prime}\right)>\alpha_{t}$, since condition V2 does not hold. Hence, $\phi_{t}(g) \geq \alpha_{t}$. If $g$ does not terminate, then $\phi_{t}(g) \geq \alpha_{t}$ follows from $\alpha_{t} \leq 0$. This shows that $t$ is $\alpha$-safe at $s$.

We say that the plan $g=\left(t_{m}\right)_{m \in \mathbb{N}}$ is $\alpha$-safe if

S1. $g$ is $\alpha$-viable;

S2. for all $m \in N$ such that $t_{m} \neq t_{m+1}$, it holds that $t_{m}$ is $\alpha$-safe at $t_{m+1}$;

S3. if $g$ terminates at $s$, then $s$ is $\alpha$-positive or $s$ is $\alpha$-vulnerable. 
We say that $\alpha \in \Omega$ is stable if for every $t \in N$, an $\alpha$-safe plan for $t$ exists. The following lemma demonstrates that stable vectors exist.

Lemma 2 The vector $\rho=\left(r_{t}(t)\right)_{t \in N}$ is stable.

Proof. Trivially, we have $\rho \in \Omega$. Let $t \in N$. Notice that termination is $\rho$-viable for $t$. So, if $t$ is $\rho$-positive or if $t$ is $\rho$-vulnerable, we choose the plan $(t, t, \ldots)$. Otherwise, $\rho_{t} \leq 0$ and $t$ is not $\rho$-vulnerable. Take an arbitrary $u \in N \backslash\{t\}$. By lemma 1.2, $t$ is $\rho$-safe at $u$. Therefore, if $u$ is $\rho$-positive or if $u$ is $\rho$-vulnerable, $(t, u, u, \ldots)$ is the required plan. Otherwise, $\rho_{u} \leq 0$ and $u$ is not $\rho$-vulnerable. By lemma 1.2 once more, $u$ is $\rho$-safe at $t$, and therefore $(t, u, t, u, \ldots)$ is the required plan.

\section{Proof of the main result}

In this section, we first provide a method for updating a stable vector $\alpha \in \Omega$, such that the updated vector, say $\delta(\alpha)$, is also a stable vector in $\Omega$, and satisfies $\delta(\alpha) \geq \alpha$ coordinatewisely. This will imply the existence of a stable vector $\alpha^{*} \in \Omega$ with the property $\delta\left(\alpha^{*}\right)=\alpha^{*}$. This property ensures that a deviation, say by player $t$, from a prescribed $\alpha^{*}-$ viable plan $g$ can be answered by prescribing a new $\alpha^{*}$-viable plan $g^{\prime}$ such that $\phi_{t}\left(g^{\prime}\right) \leq \alpha_{t}^{*}$. If the prescribed plans during play do not terminate or terminate at $\alpha^{*}$-positive states, this actually constitutes a subgame perfect 0 -equilibrium. However, if termination at a state $s$ with $\alpha_{s}^{*}<0$ is precribed, it may be necessary to have a secondary threat plan available

that is played with small probability $\epsilon$ to enforce termination at $s$. Now, the properties of $\alpha^{*}$ will be shown to be such that $s$ is $\alpha^{*}$-vulnerable, and hence an appropriate threat plan is indeed available.

For $t, u \in N$ with $u \neq t$, and $\alpha \in \Omega$, we define

$$
\begin{aligned}
& \beta_{t}^{+}(u, \alpha)=\min \left\{\phi_{t}(v) \mid v \in V_{u}(\alpha)\right\}, \\
& \beta_{t}^{-}(u, \alpha)=\min \left\{\phi_{t}(v) \mid v \in V_{u}(\alpha) \text { and } v \text { does not terminate at } t\right\},
\end{aligned}
$$

and further

$$
\begin{aligned}
& \beta_{t}(u, \alpha)= \begin{cases}\beta_{t}^{-}(u, \alpha) & \text { if } \alpha_{t} \leq 0 \text { and } t \text { is not } \alpha \text {-vulnerable } \\
\beta_{t}^{+}(u, \alpha) & \text { otherwise }\end{cases} \\
& \beta_{t}(t, \alpha)=r_{t}(t)
\end{aligned}
$$

Finally, for $t \in N$ and $\alpha \in \Omega$, we define

$$
\delta_{t}(\alpha)=\max \left\{\beta_{t}(u, \alpha) \mid u \in N\right\},
$$

and

$$
B_{t}(\alpha)=\left\{u \in N \mid \beta_{t}(u, \alpha)=\delta_{t}(\alpha)\right\} .
$$

We use the convention $\min \emptyset=\infty$, so that $\beta_{t}(u, \alpha)$ and $\delta_{t}(\alpha)$ are well defined. Moreover, by the definition of $\delta_{t}(\alpha)$, the set $B_{t}(\alpha)$ is always nonempty. 
Lemma 3 Let $\alpha \in \Omega$ be stable and let $t \in N$. Then $\alpha_{t} \leq \delta_{t}(\alpha)<\infty$.

Proof. First we show that $\alpha_{t} \leq \delta_{t}(\alpha)$. Since $\alpha$ is stable, an $\alpha$-safe plan for $t$ exists. If $(t, t, \ldots)$ is an $\alpha$-safe plan for $t$, then $\alpha_{t}=r_{t}(t)=\beta_{t}(t, \alpha) \leq \delta_{t}(\alpha)$. Otherwise, $u \in N \backslash\{t\}$ exists such that $t$ is $\alpha$-safe at $u$, which implies

$$
\alpha_{t} \leq \min \left\{\phi_{t}(v) \mid v \in V_{u}(\alpha)\right\} \leq \beta_{t}(u, \alpha) \leq \delta_{t}(\alpha)
$$

Now we prove that $\delta_{t}(\alpha)<\infty$. Choose $u \in B_{t}(\alpha)$. If $u=t$, then $\delta_{t}(\alpha)=\beta_{t}(t, \alpha)=$ $r_{t}(t)<\infty$. So assume further that $u \neq t$. Let $v$ denote an $\alpha$-safe plan for $u$. If $\alpha_{t}>0$ or if $t$ is $\alpha$-vulnerable, then $\delta_{t}(\alpha)=\beta_{t}^{+}(u, \alpha) \leq \phi_{t}(v)<\infty$. Otherwise, if $\alpha_{t} \leq 0$ and $t$ is not $\alpha$-vulnerable, then by definition of an $\alpha$-safe plan, $v$ does not terminate at $t$. Thus $\delta_{t}(\alpha)=\beta_{t}^{-}(u, \alpha) \leq \phi_{t}(v)<\infty$.

Notice that if $\delta_{t}(\alpha)<\infty$ holds for some $\alpha \in \Omega$ and $t \in N$, then $\beta_{t}(u, \alpha)<\infty$ for every $u \in N$. Hence, lemma 3 has the following corollary.

Corollary 4 Let $\alpha \in \Omega$ be stable. Then, $V_{u}(\alpha)$ is non-empty for every $u \in N$. Moreover, if for $t \in N$ it holds that $\alpha_{t} \leq 0$ and $t$ is not $\alpha$-vulnerable, then for every $u \in N$ with $u \neq t$ there exists a plan $v \in V_{u}(\alpha)$ that does not terminate at $t$.

Lemma 5 Let $\alpha \in \Omega$ be stable, let $t \in N$, and let $\delta$ be the vector that results from $\alpha$ when coordinate $\alpha_{t}$ is replaced with $\delta_{t}(\alpha)$. Then

1. $V_{u}(\delta) \subseteq V_{u}(\alpha)$ for all $u \in N$.

2. If $s, u \in N$ with $s \neq t$, and if $s$ is $\alpha$-safe at $u$, then $s$ is $\delta$-safe at $u$.

Proof. By lemma 3, we have $\delta \geq \alpha$. Then, if a plan is $\delta$-viable, it is obviously also $\alpha$-viable. This proves part 1 .

Let $s, u \in N$ with $s \neq t$, and assume that $s$ is $\alpha$-safe at $u$. Choose an arbitrary plan $g \in V_{u}(\delta)$. Then $g \in V_{u}(\alpha)$ by part 1 , and since $s$ is $\alpha$-safe at $u$, it follows that $\phi_{s}(g) \geq \alpha_{s}$. Since $s \neq t$, we have $\alpha_{s}=\delta_{s}$, hence $\phi_{s}(g) \geq \delta_{s}$. This proves part 2 .

Lemma 6 Let $\alpha \in \Omega$ be stable, let $t \in N$, and let $\delta$ be the vector that results from $\alpha$ when coordinate $\alpha_{t}$ is replaced with $\delta_{t}(\alpha)$. Then $\delta$ is stable.

Proof. We may assume $\delta_{t}>\alpha_{t}$, as otherwise the lemma is trivially true. To prove the lemma, we need to demonstrate the existence of a $\delta$-safe plan for every $u \in N$. In part A of the proof we show how a $\delta$-safe plan for $t$ can be constructed, and in part B we give a construction for $u \neq t$.

A: Choose $u \in B_{t}(\alpha)$. Notice that the assumption $\delta_{t}>\alpha_{t}$ implies $t \notin B_{t}(\alpha)$, hence $u \neq t$. We first show that $t$ is $\delta$-safe at $u$. To see this, let $g$ be a $\delta$-viable plan for $u$ (if there is no such plan, then $t$ is $\delta$-safe at $u$ by definition). If $t \in g$, then $\phi_{t}(g) \geq \delta_{t}$ follows by the 
$\delta$-viability of $g$. If $t \notin g$, then obviously $g$ does not terminate at $t$. Since $g$ is also $\alpha$-viable, it follows that $\phi_{t}(g) \geq \beta_{t}^{-}(u, \alpha) \geq \beta_{t}(u, \alpha)=\delta_{t}$. So indeed, $t$ is $\delta$-safe at $u$.

Since $\alpha$ is stable, there exists an $\alpha$-safe plan $g$ for $u$. We now argue that $g$ does not terminate at $t$. Suppose by way of contradiction that $g$ terminates at $t$. Then, since $g$ is an $\alpha$-safe plan, $\alpha_{t}>0$ or $t$ is $\alpha$-vulnerable. Hence, $\delta_{t}=\beta_{t}^{+}(u, \alpha) \leq \phi_{t}(g)=r_{t}(t)$, which contradicts our assumption that $\delta_{t}>\alpha_{t}$. Thus, $g$ does not terminate at $t$ as claimed. We distinguish two cases.

A1: $g$ terminates at some $s \neq t$. In this case, we prove that $g^{\prime}:=(t, u, s, s, \ldots)$ is a $\delta$-safe plan.

Proof of condition S1: We have $\phi_{t}\left(g^{\prime}\right)=\phi_{t}(g) \geq \beta_{t}(u, \alpha)=\delta_{t}$, and by the $\alpha$-viability of $g$, we also have $\phi_{u}\left(g^{\prime}\right)=\phi_{u}(g) \geq \alpha_{u}=\delta_{u}$ and similarly $\phi_{s}\left(g^{\prime}\right) \geq \delta_{s}$. Hence, S1 holds indeed. Proof of condition S2: We know already that $t$ is $\delta$-safe at $u$. Thus, if $u=s$ then S2 holds. If $u \neq s$, then by lemmas 1.1 and $5.2, u$ is $\delta$-safe at $s$, and S2 holds in this case as well. Proof of condition S3: As $g$ is $\alpha$-safe, we have that $s$ is $\alpha$-terminating, and moreover either $\alpha$-positive or $\alpha$-vulnerable. If $s$ is $\alpha$-positive, then $s$ is $\delta$-positive too, and S3 holds. So, assume that $s$ is $\alpha$-vulnerable. First suppose that $s$ is $\alpha$-vulnerable due to V1, so that there exists a plan $v \in V_{y}(\alpha)$ for some $y \in N \backslash\{s\}$ such that $v$ terminates at $s$ and there is an $x \in v, x \neq s$ with $r_{s}(x) \leq \alpha_{s}$. The plan $(x, s, s, \ldots)$ when $x \neq t$ and respectively the plan $g^{\prime}$ when $x=t$ show that $s$ is $\delta$-vulnerable (due to V1 in both cases). Finally, assume that $s$ is $\alpha$-vulnerable due to V2, so that an $\alpha$-terminating state $x \in N \backslash\{s\}$ exists with $r_{s}(x) \leq \alpha_{s}$. If $x \neq t$, then $s$ is $\delta$-vulnerable (due to V2), and if $x=t$, then the plan $g^{\prime}$ shows that $s$ is $\delta$-vulnerable (due to V1).

A2: $g$ does not terminate. In this case, $g^{\prime}:=(t, g)$ is $\delta$-safe when $t \notin g$, and $g^{\prime \prime}=$ $(t, u, t, u, \ldots)$ is $\delta$-safe when $t \in g$. Indeed, condition S1 holds for either case because $\delta_{t}=\beta_{t}(u, \alpha) \leq \phi_{t}(g)=0$, and because $g$ is $\alpha$-viable. Condition S2 is valid due to $t$ being $\delta$-safe at $u$, and additionally to lemma 5.2 for $g^{\prime}$ and to lemmas 5.2 and 1.1 for $g^{\prime \prime}$. Condition S3 needs no verification.

B: We construct a $\delta$-safe plan for $u$ with $u \neq t$ as follows. If $u$ is $\delta$-safe at $t$, then choose a $\delta$-safe plan $\tilde{v}$ for $t$ and $(u, \tilde{v})$ is the required $\delta$-safe plan for $u$. So assume from here that $u$ is not $\delta$-safe at $t$. Let $v$ denote an $\alpha$-safe plan for $u$. We claim that $v$ is the required $\delta$-safe plan. Observe that $t \notin v$ : otherwise $u$ is $\alpha$-safe at $t$ by lemma 1.1, hence $u$ is $\delta$-safe at $t$ by lemma 5.2, contradicting our assumption that $u$ is not $\delta$-safe at $t$. Therefore $\alpha_{x}=\delta_{x}$ for all $x \in v$, which shows that $v$ is $\delta$-viable, so condition $\mathrm{S} 1$ holds for $v$ and $\delta$. Also, $v$ consists entirely of $\delta$-safe moves by lemma 5.2 , so condition $\mathrm{S} 2$ also holds for $v$ and $\delta$. Condition S3 for $v$ and $\delta$ is satisfied when $v$ does not terminate, or when $v$ terminates at an $\alpha$-positive state. So assume that $v$ terminates at $s$ such that $\alpha_{s} \leq 0$. Since $v$ is $\alpha$-safe, $s$ must be $\alpha$-vulnerable. Notice that $s$ is not $\delta$-safe at $t$ : if $s=u$ then $s$ is not $\delta$-safe at $t$ due to our assumption that $u$ is not $\delta$-safe at $t$, whereas if $s \neq u$ then $s$ is not $\delta$-safe at $t$ because $u$ is $\delta$-safe at $s$ and $u$ is not $\delta$-safe at $t$, and because of the transitivity of safe moves (cf. lemma 1.1). Hence, $g \in V_{t}(\delta)$ exists with $\phi_{s}(g)<\delta_{s}=\alpha_{s} \leq 0$. Notice that $g$ must terminate, since $\phi_{s}(g) \neq 0$, and $g$ does not terminate at $s$, since $g$ is $\delta$-viable and $\phi_{s}(g)<\delta_{s}$. This implies that a $\delta$-terminating state $x \in N \backslash\{s\}$ exists with $r_{s}(x)<\delta_{s}$. It follows that $s$ is $\delta$-vulnerable, and condition S3 for $v$ and $\delta$ is satisfied. So indeed, $v$ is the required plan. 
Lemma 7 A stable vector $\alpha^{*} \in \Omega$ exists such that $\delta_{t}\left(\alpha^{*}\right)=\alpha_{t}^{*}$ for all $t \in N$. Moreover, for all $t \in N$, we have

$$
\alpha_{t}^{*}<0 \Rightarrow t \text { is } \alpha^{*} \text {-vulnerable. }
$$

Proof. For any $\alpha \in \Omega$, we denote by $N(\alpha)$ the set of states $t \in N$ for which $\delta_{t}(\alpha)>\alpha_{t}$. Let $\alpha^{0}=\left(r_{t}(t)\right)_{t \in N}$. By lemma 2, the vector $\alpha^{0}$ is stable. If $N\left(\alpha^{0}\right)=\emptyset$, then define $\alpha^{*}=\alpha^{0}$. Otherwise, choose an arbitrary $t^{0} \in N\left(\alpha^{0}\right)$, and let $\alpha^{1}$ be the vector that results from $\alpha^{0}$ when coordinate $\alpha_{t^{0}}^{0}$ is replaced with $\delta_{t^{0}}\left(\alpha^{0}\right)$. In view of lemmas 3 and $6, \alpha^{1} \in \Omega$ and $\alpha^{1}$ is stable too, and it is in one coordinate strictly larger than $\alpha^{0}$, while in all other coordinates they are equal. If $N\left(\alpha^{1}\right)=\emptyset$, then define $\alpha^{*}=\alpha^{1}$. Otherwise, we can increase $\alpha^{1}$ in one coordinate, just as above, and so on. Since the set of payoffs is finite, lemma 3 guarantees that, in a finite number of steps, we obtain a stable vector $\alpha^{*} \in \Omega$ such that $\delta_{t}\left(\alpha^{*}\right)=\alpha_{t}^{*}$ for all $t \in N$.

Assume that $\alpha_{t}^{*}<0$ for some $t \in N$. We need to show that $t$ is $\alpha^{*}$-vulnerable. Suppose by way of contradiction that $t$ is not $\alpha^{*}$-vulnerable. Take an arbitrary state $u \neq t$, and let $v \in V_{u}\left(\alpha^{*}\right)$ be such that $v$ does not terminate at $t$ and $\phi_{t}(v)=\beta_{t}^{-}\left(u, \alpha^{*}\right)$. Note that such a plan $v$ exists by the definition of $\beta_{t}^{-}\left(u, \alpha^{*}\right)$ and by Corollary 4 . Since $\beta_{t}^{-}\left(u, \alpha^{*}\right)=$ $\beta_{t}\left(u, \alpha^{*}\right) \leq \delta_{t}\left(\alpha^{*}\right)=\alpha_{t}^{*}<0$, the plan $v$ terminates, and by assumption, $v$ terminates at some $s \neq t$. Hence, $s$ is $\alpha^{*}$-terminating and $r_{t}(s)=\phi_{t}(v) \leq \alpha_{t}^{*}$. In view of condition V2 of vulnerability, $t$ is $\alpha^{*}$-vulnerable, which is a contradiction.

Theorem 8 A subgame-perfect $\varepsilon$-equilibrium exists, for every $\varepsilon>0$.

Proof. Fix an $\varepsilon>0$ and take a stable vector $\alpha^{*}$ as in lemma 7. Take an $R>\varepsilon$ large enough so that all payoffs in the game belong to $[-R, R]$.

Choosing plans: The most important part of the subgame-perfect $\varepsilon$-equilibrium that we will construct is the specification of how the players should react when some player $t$ deviates from a prescribed plan by nominating some player $u \neq t$. We will distinguish two types of deviations, depending on $t$ and $u$.

When a deviation of type 1 occurs, the players will react by switching to a plan $g_{t u}^{1}$ for $u$.

For a deviation of type 2 , however, we define a primary plan $g_{t u}^{2}$ as well as a secondary plan $h_{t u}^{2}$. These two plans will coincide up to a certain player $y_{t u}$. The players are expected to follow these plans until $y_{t u}$ becomes active, who is then asked to play the action corresponding to $g_{t u}^{2}$ with probability $1-\frac{\varepsilon}{2 R}$ and to play the action corresponding to $h_{t u}^{2}$ with probability $\frac{\varepsilon}{2 R}$. Subsequently, the players should execute $g_{t u}^{2}$ in the former case, and $h_{t u}^{2}$ in the latter case.

So, consider a pair $(t, u)$ where $t, u \in N$ and $u \neq t$. Choose an $\alpha^{*}$-viable plan $g$ for $u$ such that $\phi_{t}(g) \leq \alpha_{t}^{*}$. Note that such a plan exists because $\delta_{t}\left(\alpha^{*}\right)=\alpha_{t}^{*}$. If it is possible, choose $g$ such that $t \notin g$.

Case 1: We say that $(t, u)$ is of type 1 if $\alpha_{t}^{*} \geq 0$ or $t \notin g$. In this case, we define $g_{t u}^{1}=g$.

Case 2: We say that $(t, u)$ is of type 2 if $\alpha_{t}^{*}<0$ and $t \in g$. Note that in this case $g$ terminates, since $\phi_{t}(g) \leq \alpha_{t}^{*}<0$. Then in fact $g$ terminates at $t$, as otherwise a choice 
of $g$ with $t \notin g$ would have been possible, due to free transitions. Moreover, by lemma 7, player $t$ is $\alpha^{*}$-vulnerable. There are two subcases.

Case 2.1: Condition V1 is satisfied for $\alpha^{*}$ and $t$, i.e., a plan $g^{\prime} \in V_{s}\left(\alpha^{*}\right)$ exists for some $s \in N \backslash\{t\}$ such that $g^{\prime}$ terminates at $t$ and there is an $x \in g^{\prime}, x \neq t$, with $r_{t}(x) \leq \alpha_{t}^{*}$. Note that, if $s \neq u$, the plan $\left(u, g^{\prime}\right)$ is also $\alpha^{*}$-viable, since $\phi_{u}\left(\left(u, g^{\prime}\right)\right)=r_{u}(t)=\phi_{u}(g) \geq \alpha_{u}^{*}$. We may therefore assume that $g^{\prime} \in V_{u}\left(\alpha^{*}\right)$. In this subcase, we define $g_{t u}^{2}=g^{\prime}$, we define $h_{t u}^{2}$ as the plan that follows $g^{\prime}$ up to $x$ and subsequently terminates at $x$, and we define $y_{t u}=x$. (Possibly, $x=u$.)

Case 2.2: Condition V1 is not satisfied for $\alpha^{*}$ and $t$, but condition V2 is, i.e., an $\alpha^{*}$ terminating state $x \in N \backslash\{t\}$ exists such that $r_{t}(x) \leq \alpha_{t}^{*}$. In this subcase, we define $g_{t u}^{2}=g, h_{t u}^{2}=(u, x, x, \ldots)$, and $y_{t u}=u$. (Notice that $x$ cannot be the second state of $g$, since condition V1 is not satisfied. So indeed, $g_{t u}^{2}$ and $h_{t u}^{2}$ coincide up to $y_{t u}$.)

Properties of these plans: The properties below follow directly from the definitions. For every $t, u \in N$ with $u \neq t$, we have :

(A) Viability: When $(t, u)$ is of type $1, g_{t u}^{1}$ is $\alpha^{*}$-viable. When $(t, u)$ is of type $2, g_{t u}^{2}$ is $\alpha^{*}$-viable and the part of $h_{t u}^{2}$ beyond $y_{t u}$ is $\alpha^{*}$-viable, i.e., if $y_{t u}$ does not terminate, then $\phi_{s}\left(h_{t u}^{2}\right) \geq \alpha_{s}^{*}$ for every player $s$ who appears in $h_{t u}^{2}$ after $y_{t u}$.

(B) Retaliation of $t^{*}$ : When $(t, u)$ is of type $1, \phi_{t}\left(g_{t u}^{1}\right) \leq \alpha_{t}^{*}$. When $(t, u)$ is of type 2, $\phi_{t}\left(g_{t u}^{2}\right) \leq \alpha_{t}^{*}$ and $\phi_{t}\left(h_{t u}^{2}\right) \leq \alpha_{t}^{*}$.

(C) Player $t$ cannot deviate infinitely often: This is true, since for a deviation of type 1, player $t$ does not appear in $g_{t u}^{1}$, and for a deviation of type 2, player $t$ does not appear in the secondary plan $h_{t u}^{2}$.

(D) The executor of a lottery is not $t$ (the deviator) and the executer of the lottery places high probability on the plan that he prefers: When $(t, u)$ is of type $2, y_{t u} \neq t$ and $\phi_{y_{t u}}\left(g_{t u}^{2}\right) \geq \phi_{y_{t u}}\left(h_{t u}^{2}\right)$.

The profile $\sigma$ : Now we define a strategy profile $\sigma$, and afterwards we show that $\sigma$ is a subgame-perfect $\varepsilon$-equilibrium. Suppose that the initial player is $s$, and choose an arbitrary $\alpha^{*}$-viable plan $v$ for $s$. Let $\sigma$ prescribe to play as follows: Follow $v$ as long as all players choose the action prescribed by $v$. If some player $t$ deviates from $v$ by terminating, then play is strategically over. If $t$ deviates from $v$ by nominating another player $u$, then determine the type of $(t, u)$ and the corresponding plans, and play according to them as described above, until some player $t^{\prime}$ deviates by choosing an action $u^{\prime}$ that he should have used with probability zero. If $t^{\prime}$ deviates in such a way, and by doing so he terminates, i.e. $u^{\prime}=t^{\prime}$, then play is strategically over. If $u^{\prime} \neq t^{\prime}$, then just as above, determine the type of $\left(t^{\prime}, u^{\prime}\right)$ and the corresponding plans, and play according to them, until some player $t^{\prime \prime}$ deviates by choosing an action $u^{\prime \prime}$ that he should have used with probability zero, and so on.

We remark that, if $\left(t^{\prime}, u^{\prime}\right)$ is of type 2 , then player $y_{t^{\prime} u^{\prime}}$ can change the probabilities with which he chooses between two plans, without retaliation of his opponents. (Property (D) above makes sure that he cannot substantially improve his payoff by such changes.) 
The profile $\sigma$ is a subgame-perfect $\varepsilon$-equilibrium: Consider an arbitrary subgame $G(h)$, after some history $h$, and a player $t$. Notice that, according to property (A), player $t$ 's payoff without deviation would be at least

$$
\left(1-\frac{\varepsilon}{2 R}\right) \cdot \alpha_{t}^{*}+\frac{\varepsilon}{2 R} \cdot(-R) \geq \alpha_{t}^{*}-\varepsilon .
$$

We distinguish 3 cases for a single deviation by player $t$ :

Case (i): $t$ deviates in $G(h)$ by terminating. In this case, $t$ 's payoff is $r_{t}(t) \leq \alpha_{t}^{*}$, which is an improvement of at most $\varepsilon$.

Case (ii): $t$ is the player who is supposed to execute a lottery between two plans, and $t$ deviates by taking another probability distribution on the two actions that he should use. In this case, $t$ 's payoff can increase by at most $\varepsilon$ due to property (D).

Case (iii): $t$ deviates by playing a non-terminating action that he should not use at all, and by doing so he nominates player $u$. In this case, due to property (B), the new plans give player $t$ at most $\alpha_{t}^{*}$, and hence this deviation improves $t$ 's payoff by at most $\varepsilon$.

Now we examine what happens if $t$ plans to deviate once more. For this purpose, suppose that, after $t$ 's first deviation, $t$ appears again, and no termination has occurred yet. Then, $t$ is not assigned to execute a lottery. Indeed, if $t$ 's first deviation belongs to case (ii) then no new lottery is planned yet, whereas if $t$ 's first deviation belongs to case (iii) then $t$ is not assigned a new lottery due to property (D) above. Hence, $t$ 's second deviation would fall into a case similar to case (i) or case (iii) above, and $t$ 's payoff then would be at most $\alpha_{t}^{*}$. In conclusion, in view of (1), player $t$ 's improvement is still at most $\varepsilon$.

Now it follows by induction, that if $t$ decides to deviate only a finite number of times, then his payoff can only increase by $\varepsilon$.

It only remains to verify what happens if player $t$ deviates infinitely often. This of course means that play does not terminate and player $t$ receives payoff 0 . If $\alpha_{t}^{*}<0$, property (C) ensures that a plan will eventually be executed that does not contain player $t$. So, $\alpha_{t}^{*} \geq 0$ must hold. But then, by (1), player $t$ can only gain at most $0-\left(\alpha_{t}^{*}-\varepsilon\right) \leq \varepsilon$. This completes the proof.

\section{Examples}

In this section, we illustrate the construction with some examples. We start with an instructive example from Solan and Vieille [17].

Example 1. Consider the free transition game $G$ which has two players, with respective payoff-vectors

$$
r(1)=(-1,2), \quad r(2)=(-2,1) .
$$

The main idea of the game is that player 2 would like termination, and preferably at player 1 , who is not easily inclined to terminate due to his negative payoff. Solan and Vieille provided the following subgame-perfect $\varepsilon$-equilibrium $\sigma$, for $\varepsilon \in(0,1)$ : player 1 always terminates with probability 1 and player 2 always terminates with probability $\varepsilon$. Notice that player 1 is forced into termination, as trying to never terminate would eventually 
result in termination by player 2 , which would give player 1 only payoff -2 . Player 2 , on the other hand, is only playing an $\varepsilon$-best response in $\sigma$, as he could improve his payoff by $\varepsilon$ by not terminating at all. Solan and Vieille pointed out that this game does not admit a subgame-perfect 0-equilibrium.

Let us follow the iterative method given in section 3 .

Step 0: Initially, $\alpha^{0}=(-1,1)$. Note that only the plans that eventually terminate at player 1 , and $(2,2, \ldots)$ are $\alpha^{0}$-viable.

First consider player 1 . For termination we have $\beta_{1}\left(1, \alpha^{0}\right)=-1$, whereas moving to player 2 gives $\beta_{1}\left(2, \alpha^{0}\right)=-2$ due to the terminating plan $(2,2, \ldots)$. In conclusion for player 1 , $\delta_{1}\left(\alpha^{0}\right)=-1=\alpha_{1}^{0}$.

Now consider player 2. We have $\beta_{2}\left(1, \alpha^{0}\right)=2$ and $\beta_{2}\left(2, \alpha^{0}\right)=1$, hence $\delta_{2}\left(\alpha^{0}\right)=2$. So, we update player 2 in step 1 and obtain $\alpha^{1}=(-1,2)$.

Step 1: We continue with $\alpha^{1}=(-1,2)$. The only $\alpha^{1}$-viable plans for either player are the plans that eventually terminate at player 1 . It is easy to verify that $\delta_{1}\left(\alpha^{1}\right)=-1=\alpha_{1}^{1}$ and $\delta_{2}\left(\alpha^{1}\right)=2=\alpha_{2}^{1}$. This means that the iterative method terminates at step 1 , with $\alpha^{*}=\alpha^{1}=(-1,2)$. By following the construction in the proof of theorem 8 , we obtain exactly the subgame-perfect $\varepsilon$-equilibrium that Solan and Vieille found in this game.

Example 2. Consider the free transition game $G$ which has two players, with respective payoff-vectors

$$
r(1)=(-1,-2), \quad r(2)=(-2,-1) .
$$

There are two obvious subgame-perfect 0-equilibria. One is when each player always terminates with probability 1 . Another, and a more natural one, is when no player ever terminates, inducing payoff 0 to both players.

Let us follow the iterative method given in section 3 .

Step 0: Initially, $\alpha^{0}=(-1,-1)$. Note that only the following plans are $\alpha^{0}$-viable: the plans $(1,1, \ldots),(2,2, \ldots)$, and the two non-terminating plans $(1,2,1,2, \ldots)$ and $(2,1,2,1, \ldots)$.

First consider player 1 . For termination we have $\beta_{1}\left(1, \alpha^{0}\right)=-1$, whereas moving to player 2 gives $\beta_{1}\left(2, \alpha^{0}\right)=-2$ due to the terminating plan $(2,2, \ldots)$. Hence, $\delta_{1}\left(\alpha^{0}\right)=-1=\alpha_{1}^{0}$. Due to symmetry, we also obtain for player 2 that $\delta_{2}\left(\alpha^{0}\right)=-1=\alpha_{2}^{0}$.

This means that the iterative method terminates at step 0 , with $\alpha^{*}=\alpha^{0}=(-1,-1)$. By following the construction in the proof of theorem 8 , we obtain both subgame-perfect 0 -equilibria that we mentioned above (depending on the initially chosen $\alpha^{*}$-viable plan).

\section{Concluding remarks}

1. The distinction between $\beta_{t}^{+}(u, \alpha)$ and $\beta_{t}^{-}(u, \alpha)$ : Notice that, for any $t, u \in N$ satisfying $t \neq u$ and for any $\alpha \in \Omega$, we have $\beta_{t}^{+}(u, \alpha) \leq \beta_{t}^{-}(u, \alpha)$. Moreover, if $t$ is not $\alpha$-terminating, i.e., $\alpha_{t}>r_{t}(t)$, then no $\alpha$-viable plan can terminate at $t$ and hence $\beta_{t}^{+}(u, \alpha)=\beta_{t}^{-}(u, \alpha)$. Thus, regarding the iterative procedure in section 3 , the distinction between $\beta_{t}^{+}(u, \alpha)$ and $\beta_{t}^{-}(u, \alpha)$ is only relevant as long as $t$ is an $\alpha$-terminating state. 
2. Monotonicity of $\delta_{t}(\cdot)$ : The operator $\delta_{t}$ has the useful property that $\delta_{t}(\alpha) \leq \delta_{t}(\hat{\alpha})$ whenever $\alpha, \hat{\alpha} \in \Omega$ satisfy $\alpha \leq \hat{\alpha}$ in a coordinatewise sense. We will now prove this. Suppose that $\alpha \leq \hat{\alpha}$ for some $\alpha, \hat{\alpha} \in \Omega$. It suffices to prove that $\beta_{t}(u, \alpha) \leq \beta_{t}(u, \hat{\alpha})$ for every $u \in N \backslash\{t\}$. Take an arbitrary $u \in N \backslash\{t\}$. Recall that, as mentioned in the first concluding remark, $\beta_{t}^{+}(u, \alpha) \leq \beta_{t}^{-}(u, \alpha)$, and similarly for $\hat{\alpha}$. We distinguish 3 cases:

Case 1: $\beta_{t}(u, \alpha)=\beta_{t}^{+}(u, \alpha)$. In this case,

$$
\beta_{t}(u, \alpha)=\beta_{t}^{+}(u, \alpha) \leq \beta_{t}^{+}(u, \hat{\alpha}) \leq \beta_{t}(u, \hat{\alpha}) .
$$

Case $2: \beta_{t}(u, \hat{\alpha})=\beta_{t}^{-}(u, \hat{\alpha})$. In this case,

$$
\beta_{t}(u, \alpha) \leq \beta_{t}^{-}(u, \alpha) \leq \beta_{t}^{-}(u, \hat{\alpha})=\beta_{t}(u, \hat{\alpha}) .
$$

Case 3:

$$
\begin{aligned}
& \beta_{t}^{+}(u, \alpha)<\beta_{t}(u, \alpha)=\beta_{t}^{-}(u, \alpha) \\
& \beta_{t}^{+}(u, \hat{\alpha})=\beta_{t}(u, \hat{\alpha})<\beta_{t}^{-}(u, \hat{\alpha}) .
\end{aligned}
$$

We prove that this case is impossible. In view of the first concluding remark, (2) and (3) imply $r_{t}(t)=\alpha_{t}=\hat{\alpha}_{t}$. Thus, by the definition of $\beta_{t}(u, \alpha)$ and $\beta_{t}(u, \hat{\alpha})$, we may conclude that $t$ is not $\alpha$-vulnerable and that $t$ is $\hat{\alpha}$-vulnerable. Due to the latter, either V1 or V2 holds with respect to $\hat{\alpha}$.

Case 3.1: V1 holds with respect to $\hat{\alpha}$. In this case, as $V_{u}(\alpha) \supseteq V_{u}(\hat{\alpha})$ and $\alpha_{t}=\hat{\alpha}_{t}$, condition V1 also holds with respect to $\alpha$. This is a contradiction, as $t$ is not $\alpha$-vulnerable.

Case 3.2: V2 holds with respect to $\hat{\alpha}$. In this case, as $\alpha \leq \hat{\alpha}$ and $\alpha_{t}=\hat{\alpha}_{t}$, condition V2 also holds with respect to $\alpha$. This is a contradiction, as $t$ is not $\alpha$-vulnerable.

3. The uniqueness of $\alpha^{*}$ : For a free transition game $G$, let $\Delta(G)$ denote the set of stable vectors $\alpha \in \Omega$ such that $\delta_{t}(\alpha)=\alpha_{t}$ for all $t \in N$. In lemma 7 we proved, for every $G$, that $\Delta(G)$ is non-empty, which was the crucial step in the construction of a subgame-perfect $\varepsilon$-equilibrium for $G$. We remark that $\Delta(G)$ is not necessarily a singleton, as the following example demonstrates. Consider the free transition game $\hat{G}$ which has only two players, with respective payoff-vectors

$$
r(1)=(1,2), \quad r(2)=(2,1) .
$$

We argue that $(1,1),(1,2) \in \Delta(\hat{G})$. First, $\alpha=(1,1)$ and $\alpha^{\prime}=(1,2)$ are both stable, because the plan $(1,1, \ldots)$ for player 1 is both $\alpha$-safe and $\alpha^{\prime}$-safe, whereas the plan $(2,1,1, \ldots)$ for player 2 is both $\alpha$-safe and $\alpha^{\prime}$-safe. Since it is straighforward that $\delta_{t}(\alpha)=\alpha_{t}$ and $\delta_{t}\left(\alpha^{\prime}\right)=\alpha_{t}^{\prime}$ for all $t=1,2$, we have $(1,1),(1,2) \in \Delta(\hat{G})$ indeed. In fact, due to symmetry, $(2,1) \in \Delta(\hat{G})$ holds as well.

Take an arbitrary free transition game $G$. All inequalities between two vectors below are meant coordinatewisely, just as the terms non-decreasing sequence and minimal element. Recall that the iterative procedure in the proof of lemma 7 produces a non-decreasing sequence of stable vectors, starting with $r=\left(r_{t}(t)\right)_{t \in N}$ and terminating with $\alpha^{*} \in \Delta(G)$. We will now show that $\alpha^{*} \leq \hat{\alpha}$ for every $\hat{\alpha} \in \Delta(G)$, which means that $\alpha^{*}$ is the minimal 
element of $\Delta(G)$. Let $\hat{\alpha} \in \Delta(G)$. Notice that if $\alpha$ is stable and $\alpha \leq \hat{\alpha}$, then $\delta_{t}(\alpha) \leq$ $\delta_{t}(\hat{\alpha})=\hat{\alpha}_{t}$ holds because of the second concluding remark. This means that the iterative procedure only generates stable vectors that are at most $\hat{\alpha}$. In particular, $\alpha^{*} \leq \hat{\alpha}$ indeed. Thus, $\alpha^{*}$ is the minimal element of $\Delta(G)$ as claimed.

As a consequence, the iterative procedure in section 3 terminates at the same $\alpha^{*}$, irrespectively of the order in which the players are updated. The only important point is that at every step a player is updated whose coordinate strictly increases by this update.

\section{Extensions.}

4.1. Subgame-perfect 0-equilibria and subgame-perfect $\varepsilon$-equilibria in pure strategies: Example 1, which is a game by Solan and Vieille, demonstrated that our main result cannot be extended in these directions.

4.2. Stationary strategies: Within the class of stationary strategies, subgame-perfect $\varepsilon$-equilibria fail to exist in certain free transition games. Consider the following example. There are 3 players, with payoff-vectors

$$
r(1)=(2,1,4), \quad r(2)=(4,2,1), \quad r(3)=(1,4,2) .
$$

It is shown in Kuipers et al. [6] that this game has no stationary subgame-perfect 0equilibrium, and similar arguments can be used to prove the non-existence of stationary subgame-perfect $\varepsilon$-equilibrium for small $\varepsilon>0$.

4.3. Games without free transitions: It is an interesting question if the main result can be extended to similar games without the assumption of free transitions. Once the players are allowed to have more than one state and have restrictions in choosing the next state, the problem becomes more difficult. Because our construction heavily relies on the transition structure of the game, it is evident that more advanced combinatorial techniques are needed to tackle such games.

5. A polynomial algorithm. It is obvious from the iterative method that $\alpha^{*}$ can be calculated in polynomial time. Subsequently, the plans $g_{t u}^{1}, g_{t u}^{2}$ and $h_{t u}^{2}$ in the proof of Theorem 8 can also be determined in polynomial time. Hence, we obtain a polynomial time algorithm whose output directly yields a subgame-perfect $\varepsilon$-equilibrium for every $\varepsilon>0$. Note that the algorithm itself is independent of $\varepsilon$.

\section{References}

[1] Bloch F. (1996): Sequential formation of coalitions in games with Externalities and Fixed Payoff Division. Games and Economic Behavior 14, 90-123.

[2] Bueno de Mesquita B. and Lalman D. (1990): Domestic opposition and foreign war. The American Political Science Review 84, 747-765.

[3] Flesch J., Kuipers J., Schoenmakers G. and Vrieze K. (2010): Subgame perfection in positive recursive games with perfect information. Mathematics of Operations Research $35,193-207$. 
[4] Flesch J., Kuipers J., Mashiah-Yaakovi A., Schoenmakers G., Solan E. and Vrieze K. (2010): Perfect-information games with lower-semi-continuous payoffs. Mathematics of Operations Research 35, 742-755.

[5] Flesch J., Kuipers J., Mashiah-Yaakovi A., Schoenmakers G., Shmaya E., Solan E. and Vrieze K. (2011): A perfect-information game without subgame-perfect epequilibria. Preprint.

[6] Kuipers J., Flesch J., Schoenmakers G. and Vrieze K. (2008): Pure subgame-perfect equilibria in free transition games. European Journal of Operational Research 199, $442-447$.

[7] Mashiah-Yaakovi A. (2009): Periodic stopping games. International Journal of Game Theory 38, 169-181.

[8] Mashiah-Yaakovi A. (2009): Subgame-perfect equilibrium in stopping games with perfect information. Preprint.

[9] Mertens J.-F. (1987): Repeated games. Proceedings of the International Congress of Mathematicians, American Mathematical Society, Providence, RI, 1528-1577.

[10] Muthoo A. (1995): On the strategic role of outside options in bilateral bargaining. Operations Research 43, 292-297.

[11] Osborne M.J. and Rubinstein A. (1990): Bargaining and markets, Academic Press.

[12] Purves R.A. and Sudderth W.D. (2010): Perfect information games with uppersemicontinuous payoffs. Preprint.

[13] Selten R. (1965): Spieltheoretische Behandlung eines Oligopolmodells mit Nachfrageträgheit. Zeitschrift für die gesamte Staatswissenschaft 121, 301-324 and 667689.

[14] Selten R. (1973): A simple model of imperfect competition, where four are few and six are many. International Journal of Game Theory 2, 141-201.

[15] Selten R. (1978): The chain store paradox. Theory and Decision 9, 127-159.

[16] Solan E. (2005): Subgame-perfection in quitting games with perfect information. Mathematics of Operations Research 30, 51-72.

[17] Solan E. and Vieille N. (2003): Deterministic multi-player Dynkin games. Journal of Mathematical Economics 39, 911-929.

[18] Thuijsman F. and Raghavan T.E.S. (1997): Perfect information stochastic games and related classes. International Journal of Game Theory 26, 403-408.

[19] Vartiainen H. (2011): Dynamic coalitional equilibrium. Journal of Economic Theory $146,672-698$.

[20] Weg E. and Zwick R. (1994): Toward the settlement of the fairness issues in ultimatum games. Journal of Economic Behavior and Organization 24, 19-34. 